\title{
Coupled Waveguide Polarization Splitter With Slightly Different Core Widths
}

\author{
Tomohide Yamazaki, Student Member, IEEE, Hideaki Aono, Junji Yamauchi, Member, IEEE, Member, OSA, and \\ Hisamatsu Nakano, Fellow, IEEE
}

\begin{abstract}
A coupler-type transverse electric/transverse magnetic (TE/TM) polarization splitter, in which a metal-loaded core is slightly widened, is analyzed using the three-dimensional beam-propagation method. Before analyzing the splitter with the slightly different core widths, the performance of a splitter with two identical cores is evaluated. The use of the two identical cores results in a high crosstalk and a low extinction ratio due to the phase mismatch between the waveguides with and without the metal for the TE mode. Therefore, the core width in the metal-loaded waveguide is slightly widened so as to restore the phase-matching condition. In contrast to the conventional splitter with the two identical cores, the present splitter is found to offer a low crosstalk of less than $-30 \mathrm{~dB}$ and a high extinction ratio of more than $30 \mathrm{~dB}$ with a coupling efficiency of more than $97 \%$ at a wavelength of $1.55 \mu \mathrm{m}$. In addition, the wideband operation is demonstrated over a wavelength range of 1.50 to $1.61 \mu \mathrm{m}$.
\end{abstract}

Index Terms-Beam-propagation method (BPM), directional coupler, metal-clad layer, polarization splitter.

\section{INTRODUCTION}

A TRANSVERSE electric/transverse magnetic (TE/TM) polarization splitter is one of the important functional devices required for polarization diversity receivers [1], [2] and filters [3], [4]. There have been many investigations of polarization splitters using a periodic multilayer [5], [6], a Y branch [7]-[9], a directional coupler [10], [11], and a multimode-interference coupler [12], [13]. Birefringent properties are usually employed to separate the TE and TM waves. For example, polarization splitting has been achieved with branching waveguides made by proton-exchange and Ti-indiffusion in $\mathrm{LiNbO}_{3}$ [7]. A polymeric polarization splitter has been demonstrated using a low-loss polymer waveguide with a buried birefringent polyimide [8]. In [11], a directional coupler-based polarization splitter has been designed and realized using silicon-on-insulator waveguides, in which geometry-induced birefringence is utilized.

The birefringent properties can also be obtained by the use of a metal line [14], [15]. In a coupler-type splitter, a metal-clad

Manuscript received August 7, 2007; revised December 28, 2007. Current version published January 28,2009 . This work was supported in part by the "University-Industry Joint Research" Project for Private Universities, matching fund subsidy from MEXT 2003-2007.

T. Yamazaki, J. Yamauchi, and H. Nakano are with the Faculty of Engineering, Hosei University, Koganei, Tokyo 184-8584, Japan (e-mail: j.yma@k. hosei.ac.jp).

H. Aono is with Sharp Corporation, Tenri, Nara 632-8567, Japan.

Digital Object Identifier 10.1109/JLT.2008.917322 layer is loaded on one of the two rib waveguides placed in parallel. Rajarajan et al. [15] evaluated the eigenmode characteristics of the splitter through the numerical analysis, and appropriately selected the rib height and the spacing between the two rib waveguides to separate the TE and TM waves. The loss of this type of splitter remains relatively low even for the TM wave, when the field is excited from the waveguide without the metal. This is attributed to the fact that the TM-mode field mainly propagates along the waveguide without the metal. As a result, this type of splitter is expected to provide high coupling efficiencies for both TE and TM modes.

From this viewpoint, we have recently studied a metal-loaded splitter consisting of silica-based embedded waveguides [16], [17], which have the advantages of low loss and easiness of fabrication [18]. To improve the splitting properties, we have employed two techniques. One is a technique for shifting the position of the metal [16]. The other is a technique for slightly widening the core width of the metal-loaded waveguide [17]. Our preliminary calculation shows that the latter technique has the possibility of offering a high extinction ratio.

In this paper, we focus our attention on the polarization splitter, in which the metal-loaded core is slightly widened. The three-dimensional beam-propagation method (BPM) is used to numerically reveal the splitting properties in detail.

Before analyzing the splitter with the slightly different core widths, we first evaluate the performance of the splitter with two identical cores. It is shown that the use of the two identical cores results in a low extinction ratio due to the phase mismatch between the waveguides with and without the metal for the TE mode.

Next, we slightly widen the core width of the metal-loaded waveguide so that the phase-matching condition may be restored for the TE mode. In contrast to the low extinction ratio of the conventional splitter with the two identical cores, the present splitter is found to offer a low crosstalk of less than $-30 \mathrm{~dB}$ and a high extinction ratio of more than $30 \mathrm{~dB}$ with a coupling efficiency of more than $97 \%$ at a wavelength of $1.55 \mu \mathrm{m}$. In addition, this splitter is found to operate over a wide wavelength range of 1.50 to $1.61 \mu \mathrm{m}$.

\section{CONFiguration AND Numerical MethoD}

Fig. 1 illustrates the basic configuration of the TE/TM polarization splitter, in which the metal is centered on the core of waveguide \#1. The field of either TE or TM mode is excited from waveguide \#2. The coupling length of the TE mode is not sensitive to the metal width due to the slight influence of the metal. In contrast, the coupling length of the TM mode is 


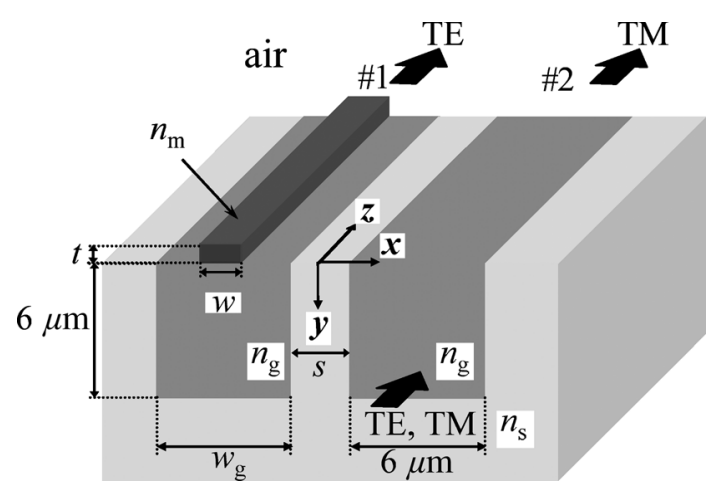

Fig. 1. Basic configuration of a TE/TM polarization splitter $(t=0.2 \mu \mathrm{m}$, $s=3 \mu \mathrm{m})$.

more sensitive to the metal width than that of the TE mode. This means that the appropriate choice of the metal width will lead to the relation of the coupling lengths, $m L_{\mathrm{TE}}=n L_{\mathrm{TM}}$, where $m$ and $n$ are integers (one of them is even and the other odd). Consequently, the TE and TM waves excited from waveguide \#2 will be extracted in waveguides \#1 and \#2, respectively. The similar operation is also obtained when the TE and TM waves are excited from waveguide \#1. It should be noted, however, that the TM wave excited from waveguide \#1 is significantly absorbed by the metal. Therefore, the input port should be waveguide \#2.

The refractive indexes of the silica-based core and substrate are set to be $n_{\mathrm{g}}=1.455$ and $n_{\mathrm{s}}=1.444$, respectively. The core width of waveguide \#2 is chosen to be $6 \mu \mathrm{m}$ and that of waveguide \#1 is denoted as $w_{\mathrm{g}}$. In this paper, the spacing between waveguides \#1 and \#2 is typically fixed to be $s=3 \mu \mathrm{m}$. Silver is used as the metal and the refractive index is taken to be $n_{\mathrm{m}}=0.14-j 11.2$ [19], [20] at a wavelength of $\lambda=1.55 \mu \mathrm{m}$. The coupling lengths of the TE and TM waves are not sensitive to a metal thickness of $t>0.1 \mu \mathrm{m}$. In addition, the TM-field absorption in the metal is almost constant for $t>0.1 \mu \mathrm{m}$. Hence, we adopt a metal thickness of $t=0.2 \mu \mathrm{m}$.

To analyze the splitter, we employ the semi-vectorial BPM using the electric field [21], [22]. The sampling widths are fixed to be $\Delta x=0.05 \mu \mathrm{m}$ and $\Delta y=\Delta z=0.01 \mu \mathrm{m}$. The perfectly matched layer is imposed at the computational window edge [23], [24].

As discussed later, we evaluate the coupling length through the eigenmode analysis using the imaginary-distance BPM [25]-[27]. The coupling length is defined by $L=\pi /\left(\beta_{\text {even }}-\beta_{\text {odd }}\right)$, where $\beta_{\text {even }}$ and $\beta_{\text {odd }}$ are the phase constants of the even and odd supermodes, respectively. The phase constant is calculated from [24, Eq. (11)].

\section{Conventional SplitTer With Two IDEntical Cores}

We first evaluate the properties of the splitter with two identical cores, i.e., $w_{\mathrm{g}}=6 \mu \mathrm{m}$. To obtain the relation of $m L_{\mathrm{TE}}=$ $n L_{\mathrm{TM}}$, we determine the metal width $w$ using the imaginarydistance BPM. Fig. 2 shows the coupling length against metal width, where the coupling length of the TM mode is multiplied by two. The coupling length of the TE mode is not sensitive to the metal width. In contrast, the coupling length of the TM

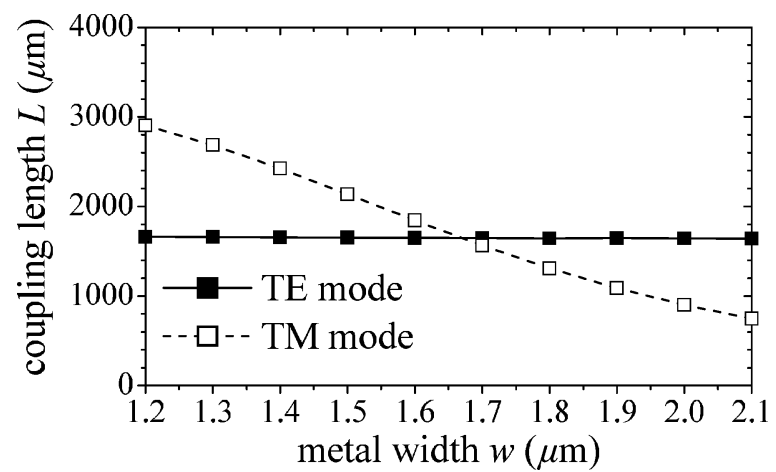

Fig. 2. Coupling length against metal width for $w_{\mathrm{g}}=6 \mu \mathrm{m}$. The coupling length of the TM mode is multiplied by two.
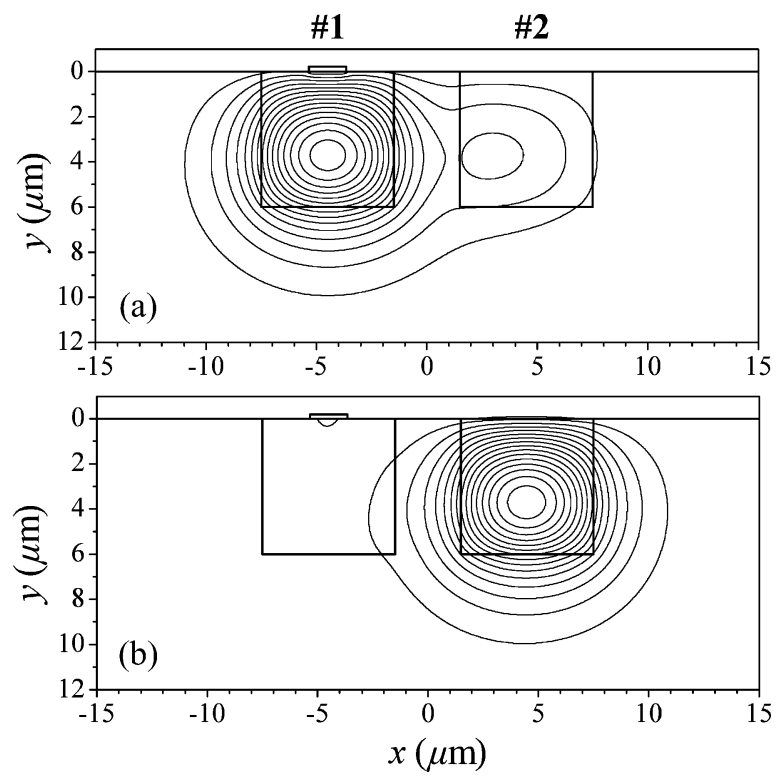

Fig. 3. Field distributions at the output for $w_{\mathrm{g}}=6 \mu \mathrm{m}$. (a) TE mode. (b) TM mode.

mode decreases as the metal width is increased, resulting in a shorter coupling length than that of the TE mode. As a result, the choice of a metal width of $1.7 \mu \mathrm{m}$ leads to the relation of $L_{\mathrm{TE}}=2 L_{\mathrm{TM}}(=1600 \mu \mathrm{m})$, which provides a minimum device length for $s=3 \mu \mathrm{m}$.

We now evaluate the splitting properties for $w=1.7 \mu \mathrm{m}$, using the propagating beam analysis. Fig. 3(a) and (b), respectively, present the field $(|E|)$ distributions of the TE and TM modes observed at the output $(z=1600 \mu \mathrm{m})$, when waveguide \#2 is excited with the eigenmode fields of isolated waveguide $\# 2$. The two polarizations are fairly separated into waveguides $\# 1$ and \#2.

Using the propagating field and the eigenmode field of the embedded waveguide without the metal, we calculate the coupling efficiencies of the TE and TM modes. The results are shown in Fig. 4. Coupling efficiencies of more than 95\% are obtained for both modes at $z=1600 \mu \mathrm{m}$. It is also observed that the TM-mode field does not sufficiently couple into waveguide \#1 at $z \approx 800 \mu \mathrm{m}$ and remains in waveguide \#2. This is because the phase constant in isolated waveguide $\# 1$ ( $\beta \approx$ 


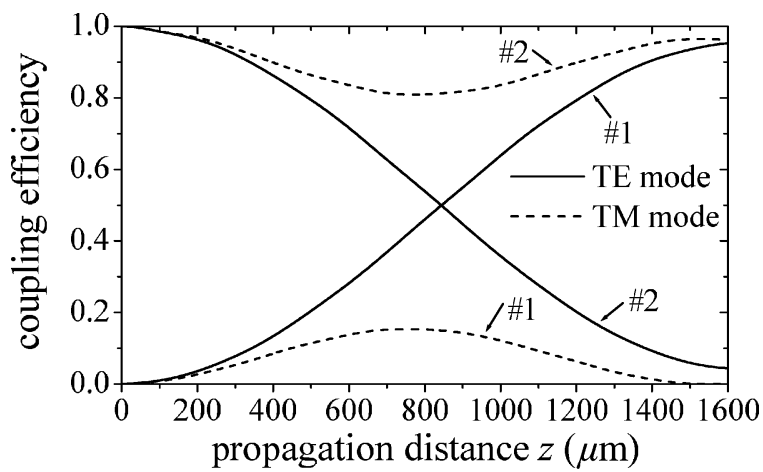

Fig. 4. Coupling efficiency against propagation distance for $w_{\mathrm{g}}=6 \mu \mathrm{m}$.

$5.877)$ is appreciably different from that in isolated waveguide $\# 2(\beta \approx 5.873)$ due to the metal effect.

A reduction in the coupling efficiency of the TM mode at the output is mainly caused by the field absorption in the metal. Note that the loss observed in Fig. 4 agrees well with that calculated from the following:

$$
\alpha=\alpha_{\text {even }} O_{\text {even }}+\alpha_{\text {odd }} O_{\text {odd }}
$$

where $O_{\text {even(odd) }}$ is the overlap integral between the even (odd) supermode field and the eigenmode field of isolated waveguide $\# 2$, and $\alpha_{\text {even(odd) }}$ the loss of the even (odd) supermode, which are obtained from the eigenmode analysis. Since the sum of the overlap integral, $O_{\text {even }}+O_{\text {odd }}$, is very close to unity, no significant transition loss is observed when waveguide \#2 is chosen as an input port.

The crosstalk and the extinction ratio are defined by

$$
\begin{aligned}
& C T_{\mathrm{TE}(\mathrm{TM})}= 10 \log _{10}\left(C_{\mathrm{TE} \# 2(\mathrm{TM} \# 1)} / C_{\mathrm{TE} \# 1(\mathrm{TM} 2)}\right) \\
& \text { for } \mathrm{TE}(\mathrm{TM}) \text { mode } \\
& E R_{\# 1(\# 2)=} 10 \log _{10}\left(C_{\mathrm{TE} \# 1(\mathrm{TM} \# 2)} / C_{\mathrm{TM} \# 1(\mathrm{TE} 2)}\right) \\
& \text { in waveguide } \# 1(\# 2)
\end{aligned}
$$

where $C$ is the coupling efficiency. The crosstalks of the TE and TM modes are calculated to be $-13 \mathrm{~dB}$ and $-28 \mathrm{~dB}$, respectively, while the extinction ratios are $28 \mathrm{~dB}$ in waveguide \#1 and $13 \mathrm{~dB}$ in waveguide \#2.

Note that the crosstalk of the TE mode is higher than that of the TM mode, and the extinction ratio in waveguide \#2 is lower than that in waveguide \#1. This is because the TE-mode field cannot sufficiently couple to waveguide \#1 and remains in waveguide \#2, as shown in Fig. 3(a). To clarify this reason, we separately evaluate the eigenmode characteristics of the TE mode in isolated waveguides \#1 and \#2. Calculation shows that the TE-mode field in isolated waveguide \#1 is somewhat deformed by the presence of the metal, i.e., the existence of the metal suppresses the extension of the field to the air region. As a result, the field is slightly extended toward the substrate. Since the refractive index of the substrate is lower than that of the core, the phase constant of waveguide $\# 1(\beta \approx 5.8732)$ becomes slightly smaller than that of waveguide $\# 2(\beta \approx 5.8735)$, leading to the phase mismatch between both waveguides. It is, therefore, desirable to increase the phase constant of waveguide \#1 and restore the phase-matching condition for the TE mode.

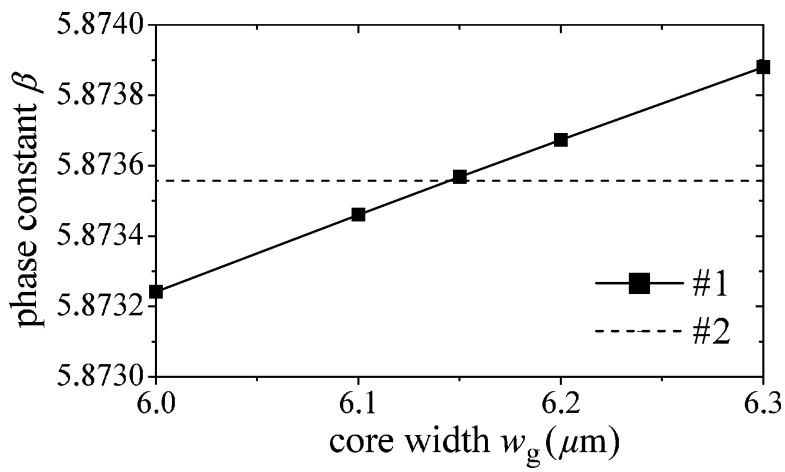

Fig. 5. Phase constant of the TE mode against core width.

\section{Modified Splitter With Slightly Different Core WIDTHS}

We now consider a technique for satisfying the phasematching condition for the TE mode. As a preliminary investigation, we intentionally increase the refractive index of the metal-loaded core (waveguide \#1). Although a slight increase in the refractive index gives rise to a slight increase in the phase constant of waveguide \#1 and restores the phase-matching condition (see the Appendix), this approach requires the tight fabrication tolerance of the core refractive index. The results shown in the Appendix, however, encourage us to employ a technique for slightly widening the core width, as a means of obtaining the comparable increase in the phase constant. Widening the core width avoids the tight fabrication tolerance of the core refractive index, while satisfying the phase-matching condition. Note that the waveguide is weakly guiding in the $x$ direction (the relative refractive-index difference is calculated to be $\Delta \simeq 0.75 \%$ between the core and substrate). Hence, slightly widening the core width will hardly deform the TE-mode field to be extracted in waveguide \#1. It follows that a reduction in the coupling efficiency is expected to be negligible between the original (6- $\mu \mathrm{m}$ width) and the widened waveguides.

Fig. 5 shows the phase constant as a function of core width. We separately calculate the phase constants of waveguides \#1 and \#2. The phase constant of waveguide \#1 is close to that of waveguide $\# 2$ over a range of $6.1 \mu \mathrm{m} \leq w_{\mathrm{g}} \leq 6.2 \mu \mathrm{m}$.

We should note that widening the core width also changes the coupling length of the TM mode slightly due to variation in the phase constants of the even and odd supermodes. The results for $w_{\mathrm{g}}=6.2 \mu \mathrm{m}$ are shown in Fig. 6, where the coupling length of the TM mode is multiplied by two. The relation of $L_{\mathrm{TE}}=2 L_{\mathrm{TM}}(=1700 \mu \mathrm{m})$ is obtained for $w=1.6 \mu \mathrm{m}$.

Since the widths of $w_{\mathrm{g}}$ and $w$ have been determined, we next assess the performance of the splitter. Fig. 7 shows the field $(|E|)$ distributions of the TE and TM modes at $z=1700 \mu \mathrm{m}$. It is observed in Fig. 7(a) that the extension of the TE-mode field into waveguide \#2 for $w_{\mathrm{g}}=6.2 \mu \mathrm{m}$ is remarkably smaller than that shown in Fig. 2(a) for $w_{\mathrm{g}}=6 \mu \mathrm{m}$.

Fig. 8 shows the coupling efficiencies against propagation distance. The present splitter maintains high coupling efficiencies of more than $99 \%$ for the TE mode and more than $97 \%$ for the TM mode around $z=1700 \mu \mathrm{m}$. It is noteworthy that as compared with the results of the splitter with the two identical 


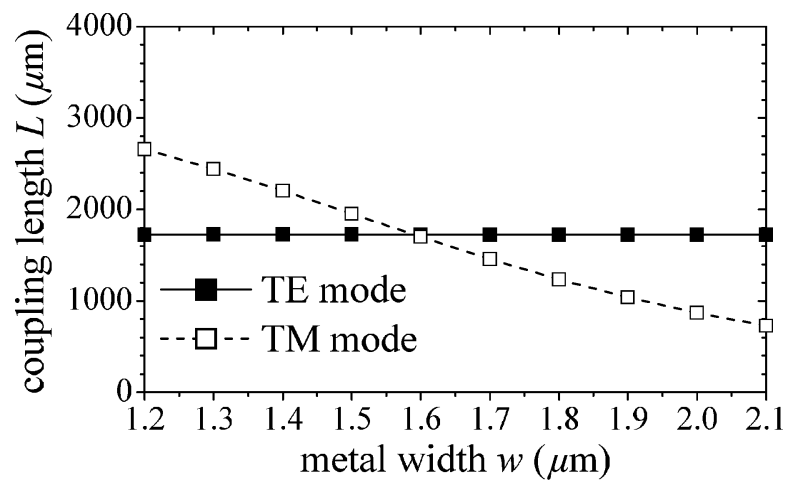

Fig. 6. Coupling length against metal width for $w_{\mathrm{g}}=6.2 \mu \mathrm{m}$. The coupling length of the TM mode is multiplied by two.
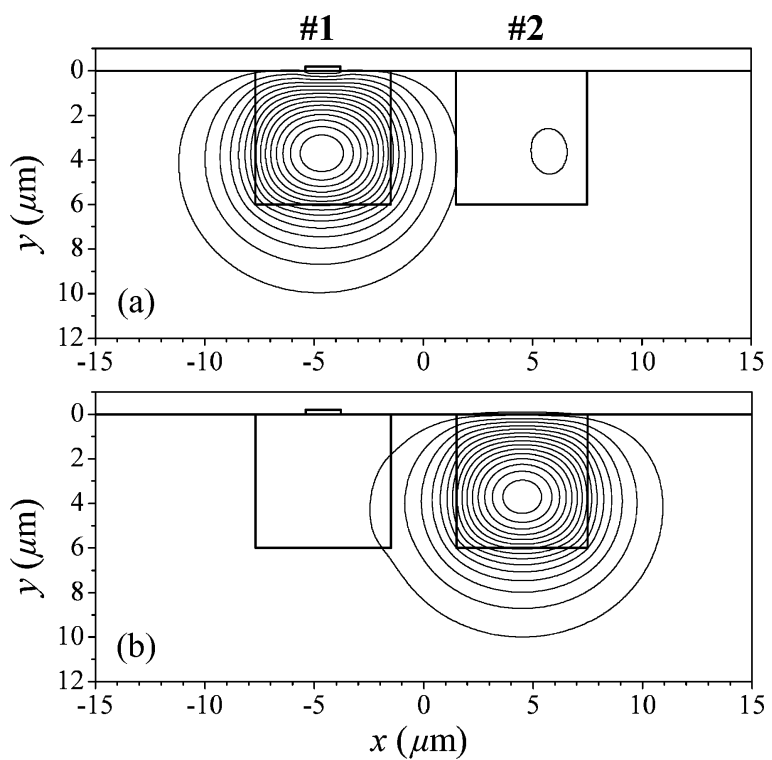

Fig. 7. Field distributions at the output for $w_{g}=6.2 \mu \mathrm{m}$. (a) TE mode and (b) TM mode.

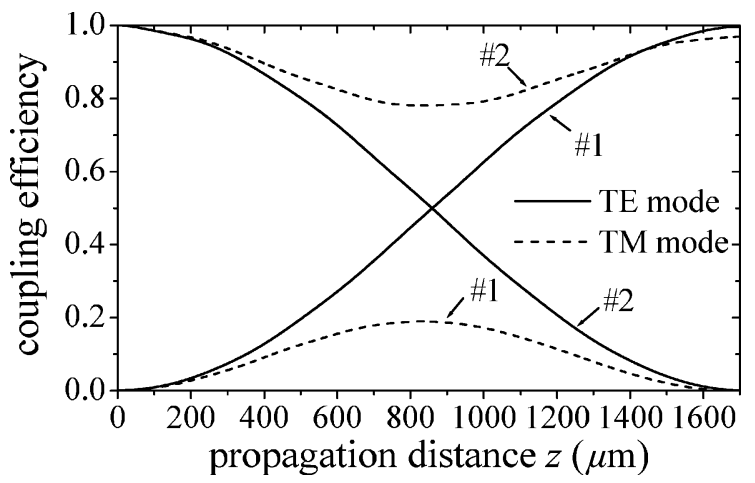

Fig. 8. Coupling efficiency against propagation distance for $w_{\mathrm{g}}=6.2 \mu \mathrm{m}$.

cores, the crosstalk of the TE mode and the extinction ratio in waveguide $\# 2$ are improved to be $-34 \mathrm{~dB}$ and $34 \mathrm{~dB}$, respectively. It is found that crosstalks of $-30 \mathrm{~dB}$ are obtained for the $\mathrm{TE}$ and TM modes and extinction ratios of $30 \mathrm{~dB}$ are obtained in waveguides \#1 and \#2. These properties are comparable to those obtained from the splitter with the two identical cores whose refractive index is slightly increased in the metal-loaded core (see the Appendix).

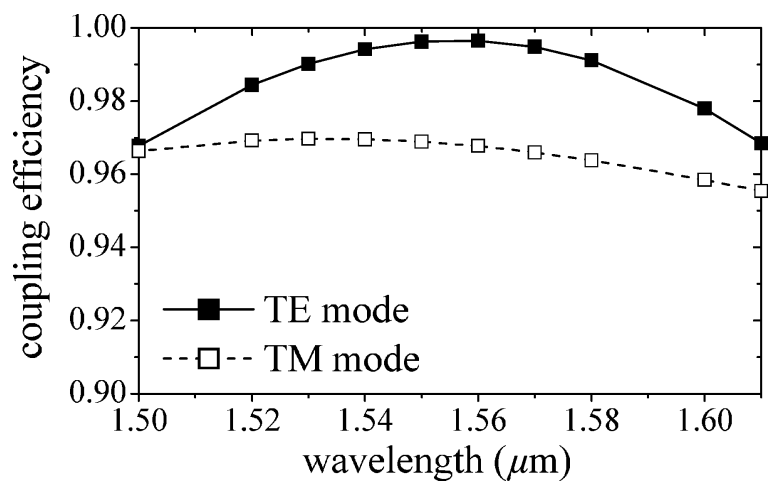

Fig. 9. Coupling efficiency against wavelength for $w_{\mathrm{g}}=6.2 \mu \mathrm{m}$.

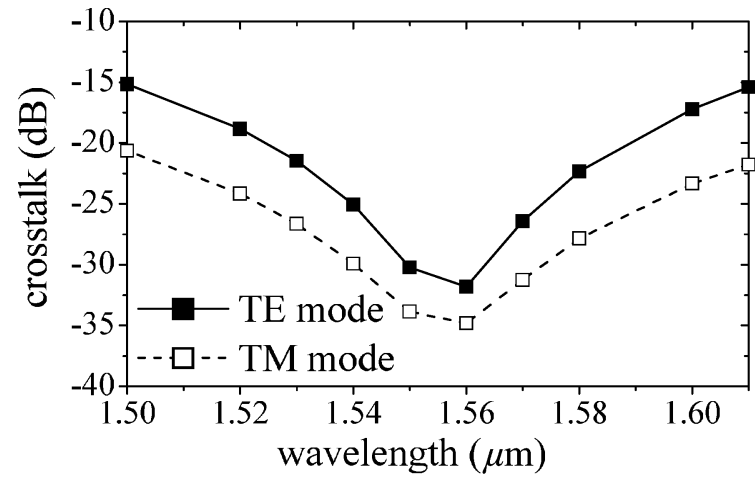

Fig. 10. Crosstalk against wavelength for $w_{\mathrm{g}}=6.2 \mu \mathrm{m}$.

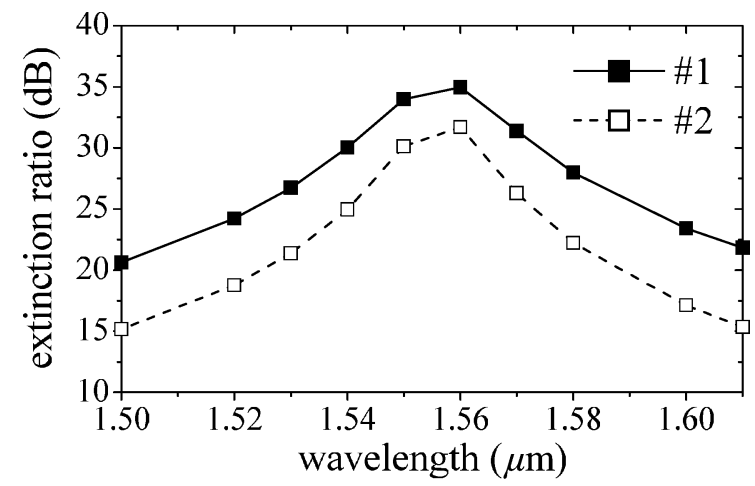

Fig. 11. Extinction ratio against wavelength for $w_{\mathrm{g}}=6.2 \mu \mathrm{m}$.

We finally evaluate the wavelength responses of the splitter. Figs. 9-11, respectively, show the coupling efficiency, crosstalk, and extinction ratio. The dispersion property of the metal refractive index is taken into account using the Drude model dielectric function [19]. A crosstalk of less than $-15 \mathrm{~dB}$ and an extinction ratio of more than $15 \mathrm{~dB}$ are obtained over a wavelength range of 1.50 to $1.61 \mu \mathrm{m}$, with the coupling efficiency being more than $95 \%$.

So far, we have neglected the propagation loss of the silicabased waveguide, which is expected to be $0.03 \mathrm{~dB} / \mathrm{mm}$ [18]. We have also analyzed the splitter with the propagation loss, and found that no significant effect is observed on the crosstalk and the extinction ratio. It should be noted, however, that the coupling efficiency is decreased in proportion to the length of the waveguide. 


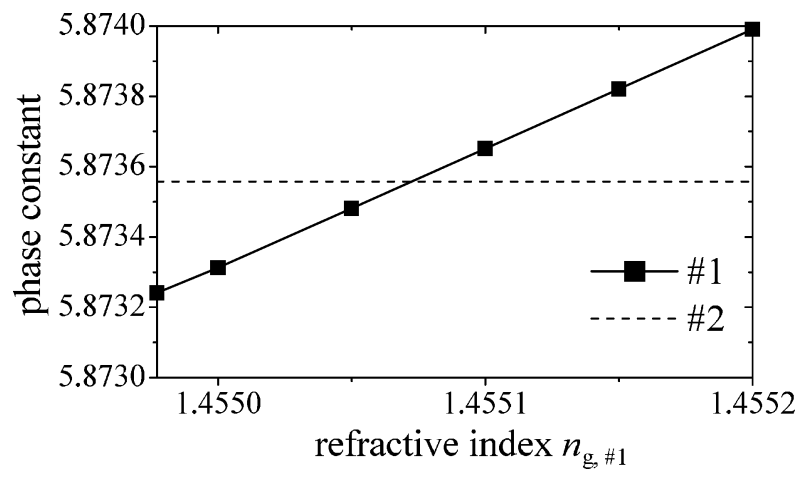

Fig. 12. Phase constant of the TE mode against refractive index of waveguide $\# 1 . w=1.7 \mu \mathrm{m}$.

In this paper, the spacing between the two parallel waveguides has been typically fixed to be $s=3 \mu \mathrm{m}$. One should note that the technique for widening the metal-loaded core is effective for any spacing (although the adjustment of $L_{\mathrm{TE}}=2 L_{\mathrm{TM}}$ is required for a specific spacing), since the phase-matching condition of the TE mode is satisfied between the isolated waveguides with and without the metal.

\section{CONCLUSION}

The three-dimensional BPM has been used to analyze a TE/TM polarization splitter consisting of silica-based embedded waveguides, in which the core width of a metal-loaded waveguide is slightly widened. Before slightly widening the core, we have assessed the performance of the conventional splitter with two identical cores. Numerical results show that the use of the two identical cores causes a low extinction ratio. This is because the phase constant of the TE mode in waveguide \#1 is slightly smaller than that in waveguide \#2, so that the TE-mode field cannot sufficiently couple to waveguide \#1 and remains in waveguide \#2. To satisfy the phase-matching condition, we slightly widen the core width in waveguide \#1. In contrast to the high crosstalk and the low extinction ratio of the conventional splitter with the two identical cores, the present splitter is found to offer a low crosstalk of less than $-30 \mathrm{~dB}$ and a high extinction ratio of more than $30 \mathrm{~dB}$ with a coupling efficiency of more than $97 \%$ at a wavelength of $1.55 \mu \mathrm{m}$. In addition, the wideband operation has been demonstrated over a wavelength range of 1.50 to $1.61 \mu \mathrm{m}$.

\section{APPENDIX}

To investigate the effect of satisfying the phase-matching condition for the TE mode, we intentionally increase the refractive index of the metal-loaded core (waveguide \#1) and evaluate the splitting properties. The core width is fixed to be $w_{\mathrm{g}}=6 \mu \mathrm{m}$. The phase constant as a function of refractive index $\left(n_{\mathrm{g}, \# 1) \text { of }}\right.$ the metal-loaded core is shown in Fig. 12. We separately calculate the phase constants of isolated waveguides \#1 and \#2. The phase constant of waveguide \#1 is close to that of waveguide \#2 over a range of $1.45505 \leq n_{\mathrm{g}, \# 1} \leq 1.4551$.

We next readjust the metal width and obtain the relation of $L_{\mathrm{TE}}=2 L_{\mathrm{TM}}$, since increasing the core refractive index slightly changes the coupling length of the TM mode due to

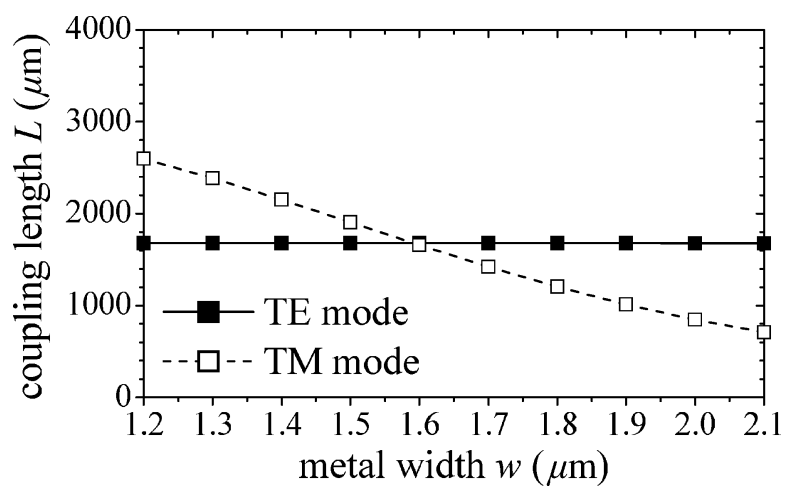

Fig. 13. Coupling length against metal width for $n_{\mathrm{g}, \# 1}=1.4551$. The coupling length of the TM mode is multiplied by two.

variation in the phase constants of the even and odd supermodes. The results for $n_{\mathrm{g}, \# 1}=1.4551$ are shown in Fig. 13 . The relation of $L_{\mathrm{TE}}=2 L_{\mathrm{TM}}(=1650 \mu \mathrm{m})$ is obtained for $w=1.6 \mu \mathrm{m}$.

Calculation through the propagating beam analysis shows that the use of a slight increase in the refractive index leads to the improvement in the splitting properties. This splitter provides a crosstalk of less than $-30 \mathrm{~dB}$ and an extinction ratio of more than $30 \mathrm{~dB}$ at a wavelength of $1.55 \mu \mathrm{m}$. It should be noted, however, that the precise control of the core refractive index is required for fabrication.

\section{REFERENCES}

[1] R. J. Deri, R. J. Hawkins, C. Caneau, E. C. M. Pennings, and N. C. Andreadakis, "Ultracompact monolithic integration of polarization diversity waveguide/photodiodes," Appl. Phys. Lett., vol. 59, no. 15, pp. 1823-1825, Oct. 1991.

[2] V. Magnin, J. Harari, and D. Decoster, "Numerical study of polarisation-selective side-illuminated pin photodetectors grown on InP substrate for hybridisation on silicon platform," Inst. Elect. Eng. Optoelectron., vol. 151, no. 3, pp. 171-176, 2004.

[3] W. Warzanskyj, F. Heismann, and R. C. Alferness, "Polarization-independent electro-optically tunable narrow-band wavelength filter," Appl. Phys. Lett., vol. 53, no. 1, pp. 13-15, Jul. 1988.

[4] T. Barwicz, M. R. Watts, M. A. Popović, P. T. Rakich, L. Socci, F. X. Kärtner, E. P. Ippen, and H. I. Smith, "Polarization-transparent microphotonic devices in the strong confinement limit," Nature Photon., vol. 1, no. 1, pp. 57-60, Jan. 2007.

[5] H. F. Mahlein, R. Oberbacher, and W. Rauscher, "An integrated optical TE-TM mode splitter," Appl. Phys., vol. 7, no. 1, pp. 15-20, May 1975.

[6] J. Yamauchi, K. Sumida, and H. Nakano, "Analysis of a polarization splitter with a multilayer filter using a Padé operator-based power-conserving fourth-order accurate beam-propagation method," IEEE Photon. Technol. Lett., vol. 18, no. 17, pp. 1858-1860, Sep. 2006.

[7] N. Goto and G. L. Yip, "A TE-TM mode splitter in $\mathrm{LiNbO}_{3}$ by proton exchange and Ti diffusion," J. Lightw. Technol., vol. 7, no. 10, pp. 1567-1574, Oct. 1989.

[8] M. C. Oh, M. H. Lee, and H. J. Lee, "Polymeric waveguide polarization splitter with a buried birefringent polymer," IEEE Photon. Technol. Lett., vol. 11, no. 9, pp. 1144-1146, Sep. 1999.

[9] T. Yamazaki, J. Yamauchi, and H. Nakano, "A branch-type TE/TM wave splitter using a light-guiding metal line," J. Lightw. Technol., vol. 25, no. 3, pp. 922-928, Mar. 2007.

[10] R. A. Betts and F. Lui, "Broadband polarisation splitting couplers in ion-exchanged glass," Electron. Lett., vol. 26, no. 7, pp. 450-452, Mar. 1990.

[11] I. Kiyat, A. Aydinli, and N. Dagli, "A compact silicon-on-insulator polarization splitter," IEEE Photon. Technol. Lett., vol. 17, no. 1, pp. 100-102, Jan. 2005.

[12] B. M. A. Rahman, N. Somasiri, C. Themistos, and K. T. V. Grattan, "Design of optical polarization splitters in a single-section deeply etched MMI waveguide," Appl. Phys. B, Photophys. Laser Chem., vol. 73 , no. 5-6, pp. 613-618, Oct. 2001. 
[13] J. M. Hong, H. H. Ryu, S. R. Park, J. W. Jeong, S. G. Lee, E. H. Lee, S. G. Park, D. Woo, S. Kim, and B. H. O, "Design and fabrication of a significantly shortened multimode interference coupler for polarization splitter application," IEEE Photon. Technol. Lett., vol. 15, no. 1, pp. 72-74, Jan. 2003.

[14] P. Albrecht, M. Hamacher, H. Heidrich, D. Hoffmann, H.-P. Nolting, and C. M. Weinert, "TE/TM mode splitters on InGaAsP/InP," IEEE Photon. Technol. Lett., vol. 2, no. 2, pp. 114-115, Feb. 1990.

[15] M. Rajarajan, C. Themistos, B. M. A. Rahman, and K. T. V. Grattan, "Characterization of metal-clad TE/TM mode splitters using the finite element method," J. Lightw. Technol., vol. 15, no. 12, pp. 2264-2269, Dec. 1997.

[16] T. Yamazaki, H. Aono, J. Yamauchi, and H. Nakano, "BPM simulation of a metal-clad TE/TM mode splitter using a silica-based embedded waveguide," in Proc. Int. Symp. Contemporary Photonic Technologies, Jan. 2005, pp. 111-112, Paper P-16.

[17] T. Yamazaki, H. Aono, J. Yamauchi, and H. Nakano, "Metal-loaded TE/TM polarization splitter consisting of embedded waveguides with slightly different core widths," presented at the Optical Society of America Integr. Photon. Res. Appl., Uncasville, CT, Apr. 26, 2006, JWB3.

[18] M. Kawachi, "Silica waveguides on silicon and their application to integrated-optic components," Opt. Quantum Electron., vol. 22, no. 5, pp. 391-416, Sep. 1990.

[19] P. B. Johnsona and R. W. Christy, "Optical constants of the noble metals," Phys. Rev. B, Condens. Matter, vol. 6, no. 12, pp. 4370-4379, Dec. 1972.

[20] S. J. Al-Bader, "Optical transmission on metallic wires-Fundamental modes," IEEE J. Quantum Electron., vol. 40, no. 3, pp. 325-329, Mar. 2004.

[21] J. Yamauchi, M. Sekiguchi, O. Uchiyama, J. Shibayama, and H. Nakano, "Modified finite-difference formula for the analysis of semivectorial modes in step-index optical waveguides," IEEE Photon. Technol. Lett., vol. 9, no. 7, pp. 961-963, Jul. 1997.

[22] J. Yamauchi, Propagating Beam Analysis of Optical Waveguides. Hertfordshire, U.K.: Research Studies Press, 2003.

[23] J. P. Berenger, "A perfectly matched layer for the absorption of electromagnetic-waves," J. Comput. Phys., vol. 114, no. 2, pp. 185-200, Oct. 1994.

[24] J. Shibayama, T. Yamazaki, J. Yamauchi, and H. Nakano, "Eigenmode analysis of a light-guiding metal line loaded on a dielectric substrate using the imaginary-distance beam-propagation method," J. Lightw. Technol., vol. 23, no. 3, pp. 1533-1539, Mar. 2005.

[25] D. Yevick and W. Bardyszewski, "Correspondence of variational finitedifference (relaxation) and imaginary-distance propagation methods for modal analysis," Opt. Lett., vol. 17, no. 5, pp. 329-330, Mar. 1992.

[26] C. L. Xu, W. P. Huang, and S. K. Chaudhuri, "Efficient and accurate vector mode calculations by beam propagation method," J. Lightw. Technol., vol. 11, no. 7, pp. 1209-1215, Jul. 1993.

[27] J. C. Chen and S. Jüngling, "Computation of higher-order waveguide modes by the imaginary-distance beam propagation method," Opt. Quantum Electron., vol. 29, no. 3, pp. S199-S205, 1994.

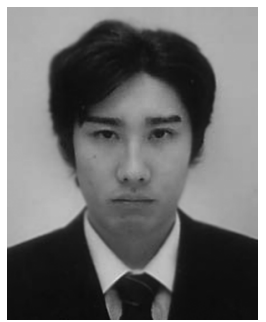

Tomohide Yamazaki (S'05) was born in Tokyo, Japan, on May 1, 1979. He received the B.E. and M.E. degrees from Hosei University, Tokyo, Japan, in 2003 and 2005, respectively, where he is currently working toward the Dr.E. degree.

Since 2005, he has been a Research Fellow of the Japan Society for the Promotion of Science. His research interests include the numerical analysis of optical waveguides.

Mr. Yamazaki is a member of the Institute of Electronics, Information and Communication Engineers

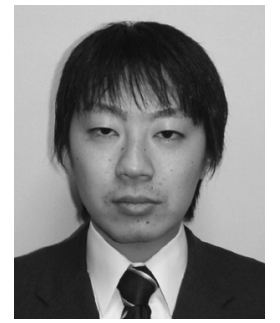

Hideaki Aono received the B.E. and M.E. degrees from Hosei University, Tokyo, Japan, in 2004 and 2006, respectively.

In 2006, he joined Sharp Corporation, Tenri, Nara, Japan. His research interests include the numerical analysis of dielectric waveguides.

Mr. Aono is a member of the Institute of Electronics, Information and Communication Engineers (IEICE) of Japan.

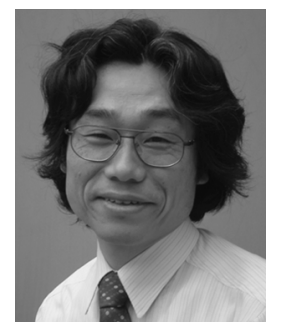

Junji Yamauchi (M'84) was born in Nagoya, Japan, on August 23, 1953. He received the B.E., M.E., and Dr.E. degrees from Hosei University, Tokyo, Japan, in 1976, 1978, and 1982, respectively.

From 1984 to 1988, he was a Lecturer with the Department of Electrical Engineering, Tokyo Metropolitan Technical College. Since 1988, he has been a member of the Faculty of Engineering, Hosei University, where he is currently a Professor of electronic informatics. He is the author of Propagating Beam Analysis of Optical Waveguides (Baldock, Hertfordshire, U.K.: Research Studies Press, 2003). His research interests include optical waveguides and circularly polarized antennas.

Dr. Yamauchi is a member of the Optical Society of America (OSA), Washington, DC and the Institute of Electronics, Information and Communication Engineers (IEICE) of Japan.

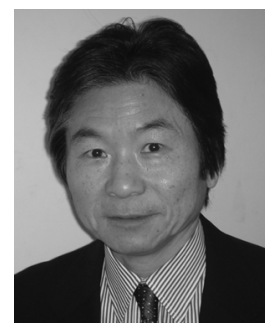

Hisamatsu Nakano (M'75-SM'87-F'92) was born in Ibaraki, Japan, on April 13, 1945. He received the B.E., M.E., and Dr.E. degrees in electrical engineering from Hosei University, Tokyo, in 1968 1970, and 1974, respectively.

Since 1973, he has been a member of the Faculty of Engineering, Hosei University, where he is currently a Professor of electronic informatics. He has published more than 200 refereed journal papers and 200 international symposium papers on antenna and relevant problems. He was a Visiting Associate Professor with Syracuse University, Syracuse, NY, during May-September 1981 a Visiting Professor with the University of Manitoba, Winnipeg, MB, Canada, during March-September 1986; and a Visiting Professor with the University of California, Los Angeles, during September 1986-March 1987. He is the author of Helical and Spiral Antennas (New York: Research Studies, Wiley, 1987) and the chapter "Antenna analysis using integral equations," in Analysis Methods of Electromagnetic Wave Problems, Vol. 2 (Norwood, MA: Artech House, 1996). His research interests include numerical methods for antennas, electromagnetics wave scattering problems, and light wave problems.

Dr. Nakano is an Associate Editor of the IEEE ANTENNAS AND PROPAGATION MAGAZINE. He was the recipient of an International Scientific Exchange Award from the Natural Sciences and Engineering Research Council of Canada, the Best Paper Award from the Inst. Elect. Eng. 5th International Conference on Antennas and Propagation in 1987, the IEEE AP-S Best Application Paper Award (H. A. Wheeler Award) in 1994, and the IEEE AP-S Chen-To Tai Distinguished Educator Award in 2006.

(IEICE) of Japan. 